\title{
Refined prediction of vertical gradient of gravity at Etna volcano gravity network (Italy)
}

\author{
Pavol ZAHOREC ${ }^{1}$, Juraj PAPČO ${ }^{2}$, Peter VAJDA $^{1}$, Filippo GRECO $^{3}$, \\ Massimo CANTARERO ${ }^{3}$, Daniele CARBONE ${ }^{3}$ \\ ${ }^{1}$ Division of Geophysics, Earth Science Institute, Slovak Academy of Sciences, \\ Bratislava, Slovak Republic; e-mail: zahorec@savbb.sk \\ ${ }^{2}$ Department of Theoretical Geodesy, Faculty of Civil Engineering, \\ Slovak University of Technology, Bratislava, Slovak Republic \\ ${ }^{3}$ Instituto Nazionale di Geofisica e Vulcanologia, Sezione di Catania, Osservatorio Etneo, \\ Catania, Italy
}

\begin{abstract}
Predicted values of the vertical gradient of gravity (VGG) on benchmarks of Etna's monitoring system, based on calculation of the topographic contribution to the theoretical free-air gradient, are compared with VGG values observed in situ. The verification campaign indicated that improvements are required when predicting the VGGs at such networks. Our work identified the following factors to be resolved: (a) accuracy of the benchmark position; (b) gravitational effect of buildings and roadside walls adjacent to benchmarks; (c) accuracy of the digital elevation model (DEM) in the proximity of benchmarks. Benchmark positions were refined using precise geodetic methods. The gravitational effects of the benchmark-adjacent walls and buildings were modeled and accounted for in the prediction. New high-resolution DEMs were produced in the innermost zone at some benchmarks based on drone-flown photogrammetry to improve the VGG prediction at those benchmarks. The three described refinements in the VGG prediction improved the match between predicted and in situ observed VGGs at the network considerably. The standard deviation of differences between the measured and predicted VGG values decreased from 36 to $13 \mu \mathrm{Gal} / \mathrm{m}$.
\end{abstract}

Key words: VGG, topographic effect, building correction, DEM, volcano gravity monitoring network

\section{Introduction}

Many active or dormant volcanoes are monitored by repeated gravity measurements aimed to detect and interpret spatiotemporal gravity changes. 
The latter can be used to study subsurface processes associated with magma dynamics, thus contributing to forecasts about possible eruptive events (e.g. Battaglia et al., 2008; Carbone et al., 2017; Fernández et al., 2017). In such time-lapse surveys the knowledge of the true (in-situ) values of the vertical gradient of gravity (VGG) plays an important role. The use of various types of gravity meters, relative and/or absolute that are fitted with sensors at different heights requires proper consideration of the VGG to "reduce" data readings to the ground or to a common level. If the VGG values are not observed in-situ, they can be modeled (predicted). The quality of this prediction is the subject of the presented work, which is focused on a case study from Etna.

The deviation of the in-situ real VGG from the normal (theoretical) value of $-308.6 \mu \mathrm{Gal} / \mathrm{m}\left(1 \mu \mathrm{Gal} / \mathrm{m}=10^{-8} \mathrm{~s}^{-2}\right)$ strongly depends on the nearby topography. A great part of this deviation can be modeled provided that a detailed digital elevation model (DEM) with sufficient resolution (accuracy), as well as correct rock-density information, is available. We have analyzed this topographic contribution to the VGG for the case of Teide volcano on Tenerife, Canary Islands (Zahorec et al., 2016). A good agreement was obtained between the calculated (predicted) and the subsequently in-situ verified VGGs on several benchmarks of the Teide gravity monitoring network. These results motivated us to conduct a similar study at Etna volcano.

The gravity monitoring network of Etna (for time-lapse measurements) consists of 71 benchmarks (Fig. 1; Carbone and Greco, 2007; Greco et al., 2012). The network is operated by INGV Osservatorio Etneo since 1986 (Budetta et al., 1989). Relative gravity surveys have been gradually complemented by 13 absolute gravity points allowing the so-called hybrid microgravity surveys (Greco et al., 2012). Several campaigns of VGG measurements have been also realized on absolute as well as relative benchmarks (Greco et al., 2012; Bonforte et al., 2017). Our primary effort was to compare the in-situ measured VGGs with our calculated (predicted) values based on the contribution of topographic masses to the gradient. Our first results showed that the match between predicted and observed VGGs was not satisfactory. Therefore, we decided to carry out a dedicated gravity campaign aimed at identifying the causes of the above misfit, at improving the calculation, and at observing some additional in-situ VGG values. 


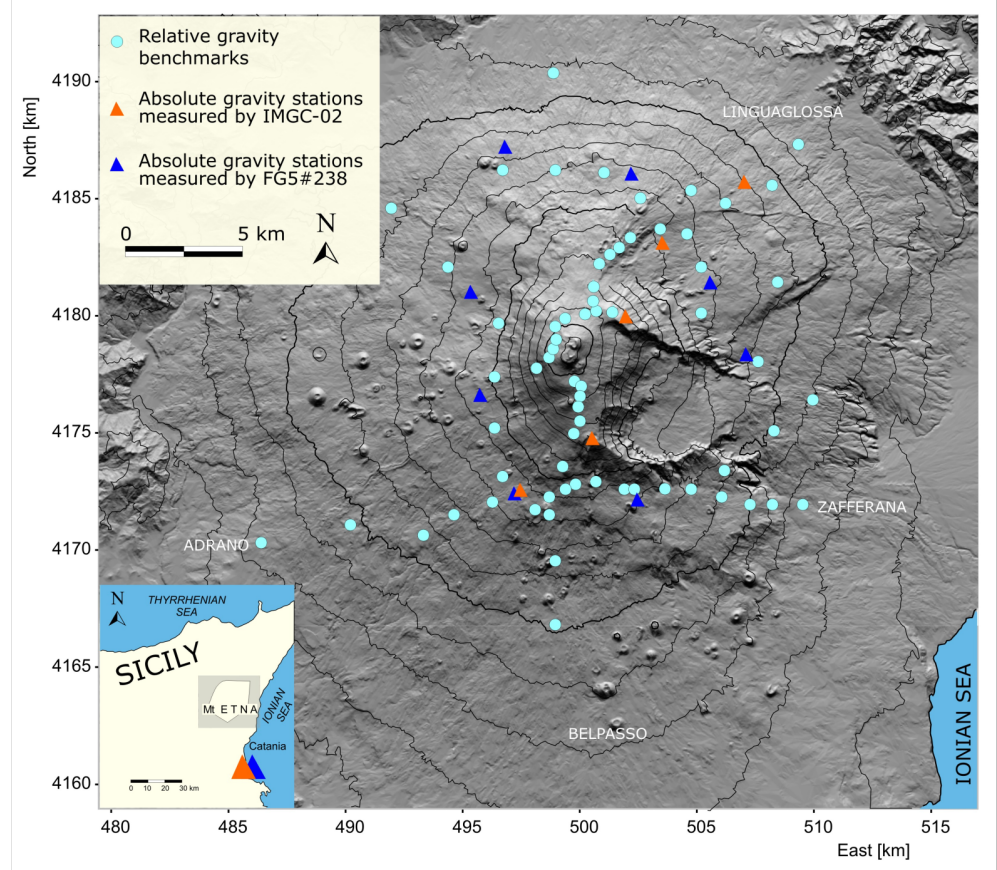

Fig. 1. Sketch map of Etna showing the benchmarks for time-lapse relative (circles) and absolute (triangles) gravity measurements. See upper left inset for explanations.

In the first part of the article we describe the method of the VGG prediction. Next, we focus on in-situ data acquisition and processing and finally we discuss the improvements to the VGG prediction.

\section{VGG prediction on the benchmarks of Etna's gravity net- work}

In previous studies (Vajda et al., 2015; Zahorec et al., 2016) it was found that in-situ measured VGGs in volcanic areas can deviate significantly from the theoretical value depending on topography conditions (besides the geology contribution which is usually less important in areas of prominent topography). We measured VGG values in the range -125 to $-506 \mu \mathrm{Gal} / \mathrm{m}$ in the Teide volcano area. Extreme deviations, low and high in absolute sense, are 
associated with rough terrain shapes: narrow valleys (gorges, canyons) and sharp convex features (peaks, ridges, caldera rims), respectively.

We predicted the VGG values by computing the topographic (and also bathymetric) contribution to the gradient. To that end, we used the proprietary software Toposk (Zahorec et al., 2017). This program was especially developed to calculate the topographic effect (i.e. the gravitational effect of the masses between the topo-surface and the zero level) at arbitrary point locations, e.g. above or below the topographic surface. This comes very handy when VGGs are calculated over benchmarks that lie below the terrain level. Such is the case of the absolute point at the Pizzi Deneri volcanological observatory (PDN; $2820 \mathrm{~m}$ a.s.l.), located in the basement of the observatory.

The topographic contribution to the VGG is simply computed as the difference between the topographic effects calculated at the heights of 1.25 and $0.25 \mathrm{~m}$, respectively, above the surface. These heights equal approximately to the CG-5 gravity meter sensor positions during the VGG measurements in the field using a tower. The topographic (and also bathymetric) effect is considered up to the standard distance of $166.7 \mathrm{~km}$, while the integration area is divided into the following zones: inner zone up to $250 \mathrm{~m}$ around the calculation point, intermediate zone $250-5240 \mathrm{~m}$ and outer zones from 5.24 up to $166.7 \mathrm{~km}$. The inner and intermediate zones play dominant roles in the contribution to the VGG. A high-precision DEM, such as those derived from LiDAR data, is required especially within the inner zone. In the presented study we used an available LiDAR-based DEM produced by Bisson et al. (2015). The DEM was produced by INGV (Sezione di Pisa), on the grounds of a survey completed in 2005. The original DEM with spatial resolution of $2 \mathrm{~m}$ (vertical accuracy of $\pm 0.24 \mathrm{~m}$ ) was resampled to $5 \mathrm{~m}$ resolution (provided in ASCII format). Final grid was computed using the Surfer software using Nearest Neighbor interpolation procedure with the grid cell size of $5 \mathrm{~m}$ (Fig. 2). Although this DEM is of relatively high quality, it does not account for morphological changes that occurred in some areas after 2005. In addition, some parts of the model feature a lower quality (due to missing LiDAR data). These facts complicate our VGG prediction. Therefore, we decided to complement the DEM through detailed drone-flown photogrammetry models in the nearest zone, wherever necessary and possible.

The topographic as well as bathymetric contributions from the outer 


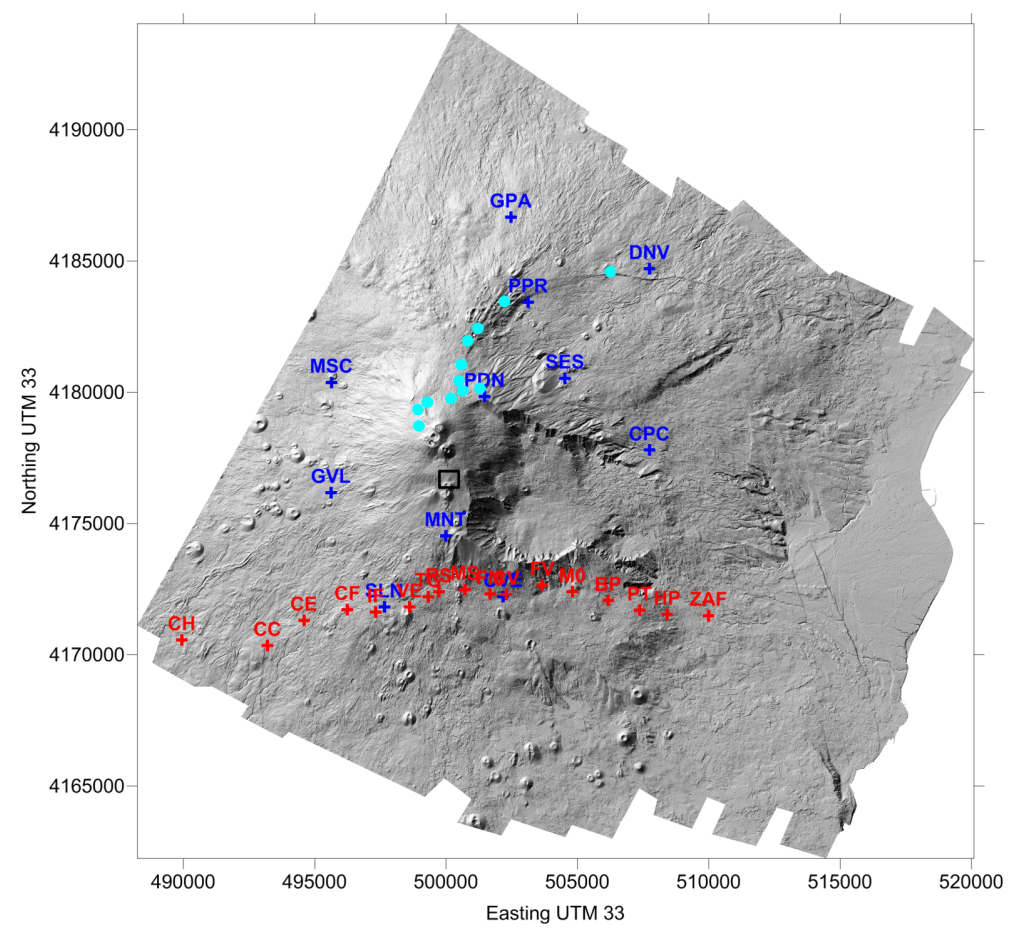

Fig. 2. Shaded relief map of the LiDAR-based DEM (Bisson et al., 2015) with the positions of measured and predicted VGG points. Absolute gravity points are blue crosses, relative benchmarks are red crosses. Cyan circles represent points along the NE-rift. The little black square encloses the cloud of points with observed VGGs near the Torre del Filosofo area (2800 $\mathrm{m}$ a.s.l.).

zones (beyond $5.24 \mathrm{~km}$ ) were calculated using SRTM data (Jarvis et al., 2008; Olson et al., 2016). The maximum topographic contribution to the VGG from the outer zones represents about $+20 \mu \mathrm{Gal} / \mathrm{m}\left(10^{-8} \mathrm{~s}^{-2}\right)$. For comparison, the maximum contribution from the inner and intermediate zones is $-176 \mu \mathrm{Gal} / \mathrm{m}$ (for the density of $2670 \mathrm{~kg} / \mathrm{m}^{3}$ ). The bathymetric contribution to the VGG (for the density of $-1640 \mathrm{~kg} / \mathrm{m}^{3}$ ) in our Etna area is negligible, it amounts to only -1 to $-2 \mu \mathrm{Gal} / \mathrm{m}$.

The correct estimation of the density of topographic masses can be a serious problem due to high rock-density variability in volcanic areas. For example, in the case of Teide volcano the adopted density of $2200 \mathrm{~kg} / \mathrm{m}^{3}$ yielded in general successful results (Zahorec et al., 2016), but with caution 
that the real density could be even considerably smaller in some places such as the areas of young slag lavas or thermally altered layers. As Etna represents a large basaltic composite volcano (Branca et al., 2011), we opted for a higher density. We have used the density of $2670 \mathrm{~kg} / \mathrm{m}^{3}$, which is in accordance with Schiavone and Loddo (200\%), who used this value as reference density for the Bouguer anomaly map of Etna.

The total (from all integration zones) estimated topographic contribution to the VGG is added to the theoretical value $-308.6 \mu \mathrm{Gal} / \mathrm{m}$ to predict the VGG at a given point. Figure 3 shows the predicted VGG values compared with the measured ones on absolute gravity points and relative benchmarks.

Figure 3 shows that the fit between measured and predicted values is generally not good (notice that some measured VGGs are scattered due to poor
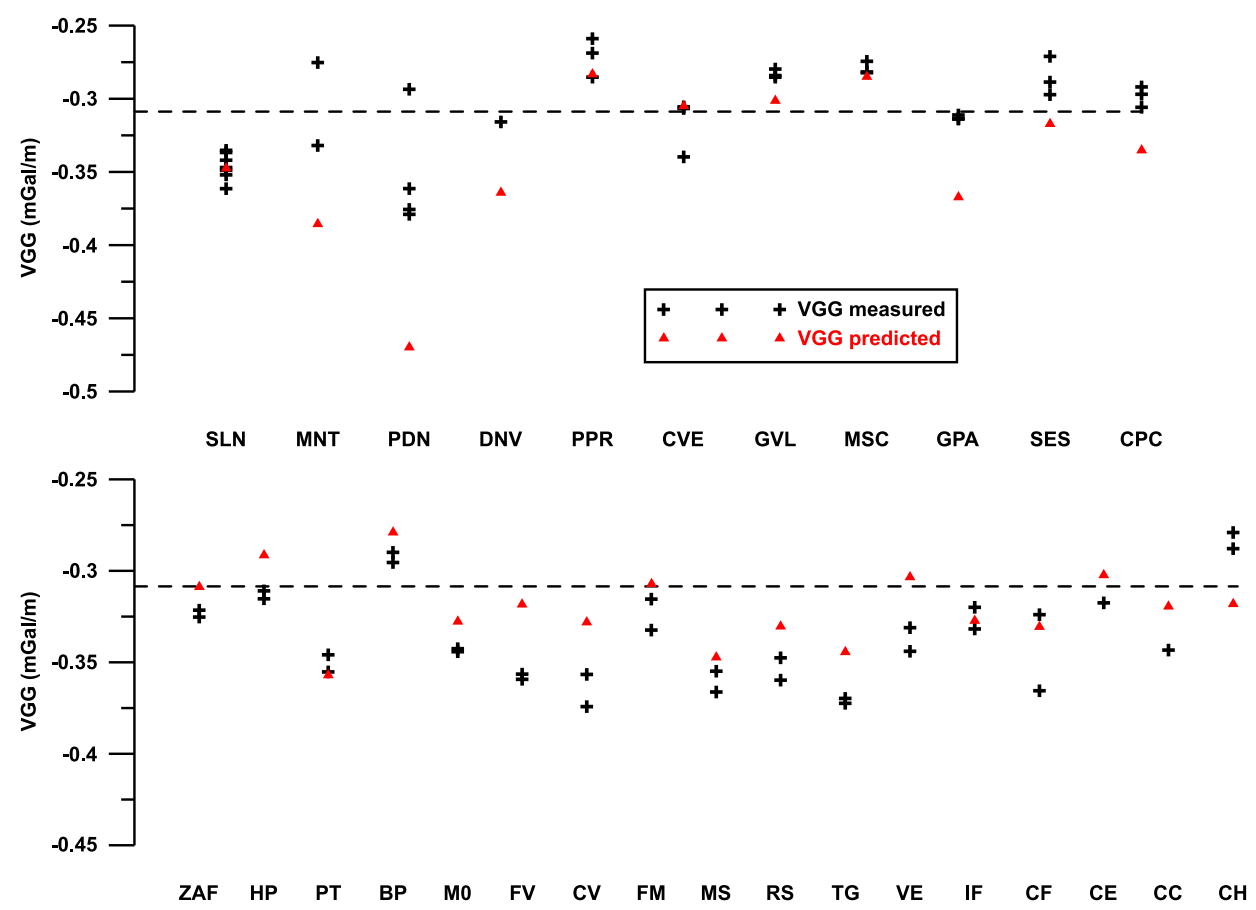

Fig. 3. Predicted VGGs (red triangles) on absolute gravity points (upper graph) and relative benchmarks (bottom graph). Black crosses represent measured VGGs. Dashed line represents theoretical (normal) VGG of $-308.6 \mu \mathrm{Gal} / \mathrm{m}$. Station positions are shown in Fig. 2. 
observation conditions). The first cause of misfit we considered was a possible station position error. We assumed this error because we knew that the stations were not positioned using precise geodetic point positioning (point coordinates were determined only by hand-held GPS). In addition, there seemed to be a systematic shift between measured and predicted VGGs. In the case of absolute gravity points a systematic shift to higher predicted values (in their absolute value) was apparent, while relative points exhibited an opposite tendency (Fig. 3). On the ground of previous observations, we hypothesized that this could be due to local positioning and near building effects (Zahorec et al., 2014). Therefore, we decided to perform additional in-situ observations, aimed at (i) verifying the position of the observation points and (ii) precisely determining the geometrical characteristics of anthropic structures (walls, buildings, etc.) in close vicinity to relative and absolute gravity points, in order to model their effect on VGG values.

We also planned to verify the VGGs measured in 2009 (Maucourant et al., 2014) along a cloud of points clustered in a small area near the 2002 eruptive vent (see little square in Fig. 2). We realized that our prediction at these points was completely inadequate (Fig. 4). Indeed the topography of this area underwent a drastic change, due to the fall-out products erupted from Etna's main craters. We mention this to illustrate that on active volcanoes large topographic changes may occur over relatively short intervals, implying that the available DEM may become inadequate in some parts shortly after its production.

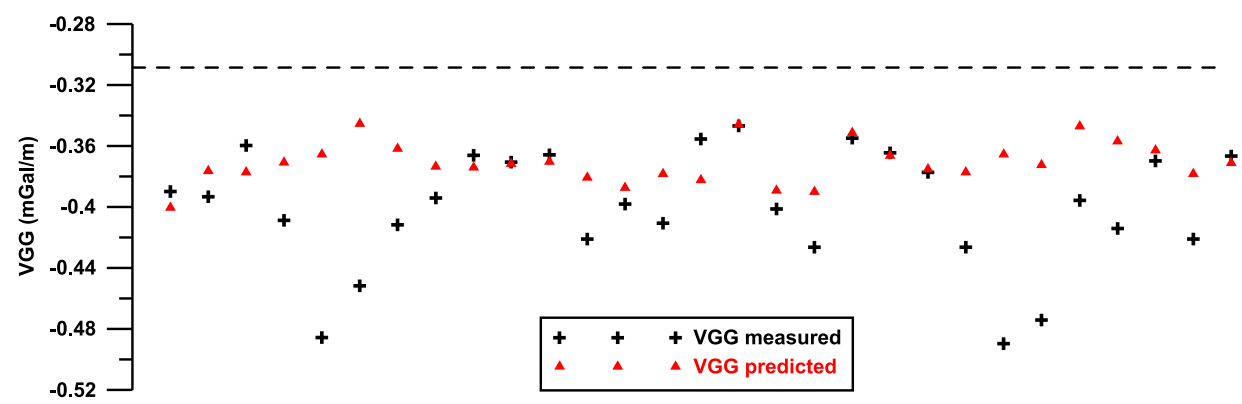

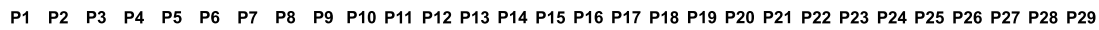

Fig. 4. Misfit between predicted (red triangles) and measured (black crosses) VGGs at Torre del Filosofo area in 2009. Dashed line represents theoretical (normal) VGG of $-308.6 \mu \mathrm{Gal} / \mathrm{m}$. 
We focused also on benchmarks of the gravity-deformation monitoring profile established in the area of the NE-rift (Fig. 2). The measurements between 2002 and 2003 captured very high vertical displacements (more than $2 \mathrm{~m}$ ) associated with the 2002-2003 NE-rift eruption, as well as gravity variations up to $350 \mu \mathrm{Gal}$ already corrected for the free-air effect using the normal free-air gradient $-308.6 \mu \mathrm{Gal} / \mathrm{m}$ (Bonforte et al., 2007). At the benchmarks of this profile the true VGG values were not known. We thus decided to assess the difference between the free-air correction based on predicted and theoretical VGGs. Since our prediction indicated VGG values quite different from normal free-air gradient on several benchmarks, a successful prediction would be of great value, particularly in this case, where a large difference between gradients is multiplied by a very large vertical displacement, see Table 1. Due to harsh observation conditions (strong wind in combination with unstable ground) in-situ measurements could be performed at only one of the NE-rift benchmarks, see next chapter.

Table 1. Expected deviation in free-air correction as a result of difference between predicted VGG and normal free-air gradient $(-308.6 \mu \mathrm{Gal} / \mathrm{m})$ multiplied by observed vertical displacement at three selected benchmarks of the NE-rift gravity-deformation monitoring profile (cyan circles in Fig. 2).

\begin{tabular}{|c|c|c|c|}
\hline Point & $\begin{array}{c}\text { Vertical } \\
\text { displacement }(\mathrm{m})\end{array}$ & $\begin{array}{c}\text { Predicted VGG } \\
(\mu \mathrm{Gal} / \mathrm{m})\end{array}$ & $\begin{array}{c}\text { Expected } \\
\text { deviation }(\mu \mathrm{Gal})\end{array}$ \\
\hline LZ & -2.16 & -371 & 135 \\
\hline DG & -2.329 & -357 & 113 \\
\hline CO & -1.403 & -374 & 92 \\
\hline
\end{tabular}

\section{In-situ geodetic and VGG observations}

In July 2018 we conducted a gravity campaign on Etna in order to make precise in-situ geodetic positioning on microgravity network points as well as to verify several VGG predictions. Our goal was to improve consistency between calculated (predicted) and in-situ measured VGGs on network benchmarks. In addition, we also carried out new VGG measurements with the same objective.

Spatial coordinates of all benchmarks, as well as of ground control points (GCP) for our surveying and photogrammetry, were determined using GNSS 
and traditional terrestrial measurements. GNSS measurements used Network and Satellite based Real Time Kinematic (NRTK/SRTK) or Fast Static Post-processing (FS) mode. NRTK measurements were realized using the Italy HEXAGON positioning service HxGN SmartNet (http://it-xpos .nrtk.eu/spiderweb/frmIndex.aspx) in Virtual Reference Station concept. SRTK measurements were realized using satellite Trimble RTX technology (https://www.trimble.com/positioning-services/trimble-rt $\mathrm{x} . \operatorname{aspx}$ ).

SRTK and Fast static method with precise post-processing was used on sites with no internet connection or with bad observation conditions (near the trees). For FS method the nearest permanent GNSS stations from Etna's geodetic network (Palano et al., 2010) or HxGN SmartNet GNSS network were used as reference points. Traditional terrestrial measurements were performed in the places without GNSS signal availability (in the buildings or in the forests). Measurements were realized with the Trimble R10 GNSS receiver and Trimble M3 total station. Average observation period was about 3 minutes in the case of the NRTK measurements, and about 20-30 minutes in the case of the SRTK or fast static measurements. Final processing was performed in Trimble Business Centre (v. 4.10) software. Spatial positions of the points were determined primarily in the ETRS89 coordinates system and then transformed to UTM33 projection. Physical heights of the points were transformed from ellipsoidal ones using global geopotential model EGM2008 (Pavlis et al., 2012). Horizontal and vertical accuracy achieved for the VGG and GCP points is better than $5 \mathrm{~cm}$.

After processing the measurements we found differences between new exact GNNS coordinates and the previous approximate positions to be in the range of 0.7 to $28 \mathrm{~m}$ in horizontal position and 0.4 to $10 \mathrm{~m}$ in vertical position.

The majority of relative benchmarks is situated on the roadside rockwalls (Fig. 5). This is for practical reasons: during the winter season the benchmarks do not get covered by the snow piled up by snow plows. The gravity effect on the gradient of these walls is not negligible, and thus it has to be modeled. Therefore we precisely measured the dimensions of the walls as well as benchmark positions with respect to the walls, and calculated their effect that was added to the nearest zone contribution in VGG prediction. 

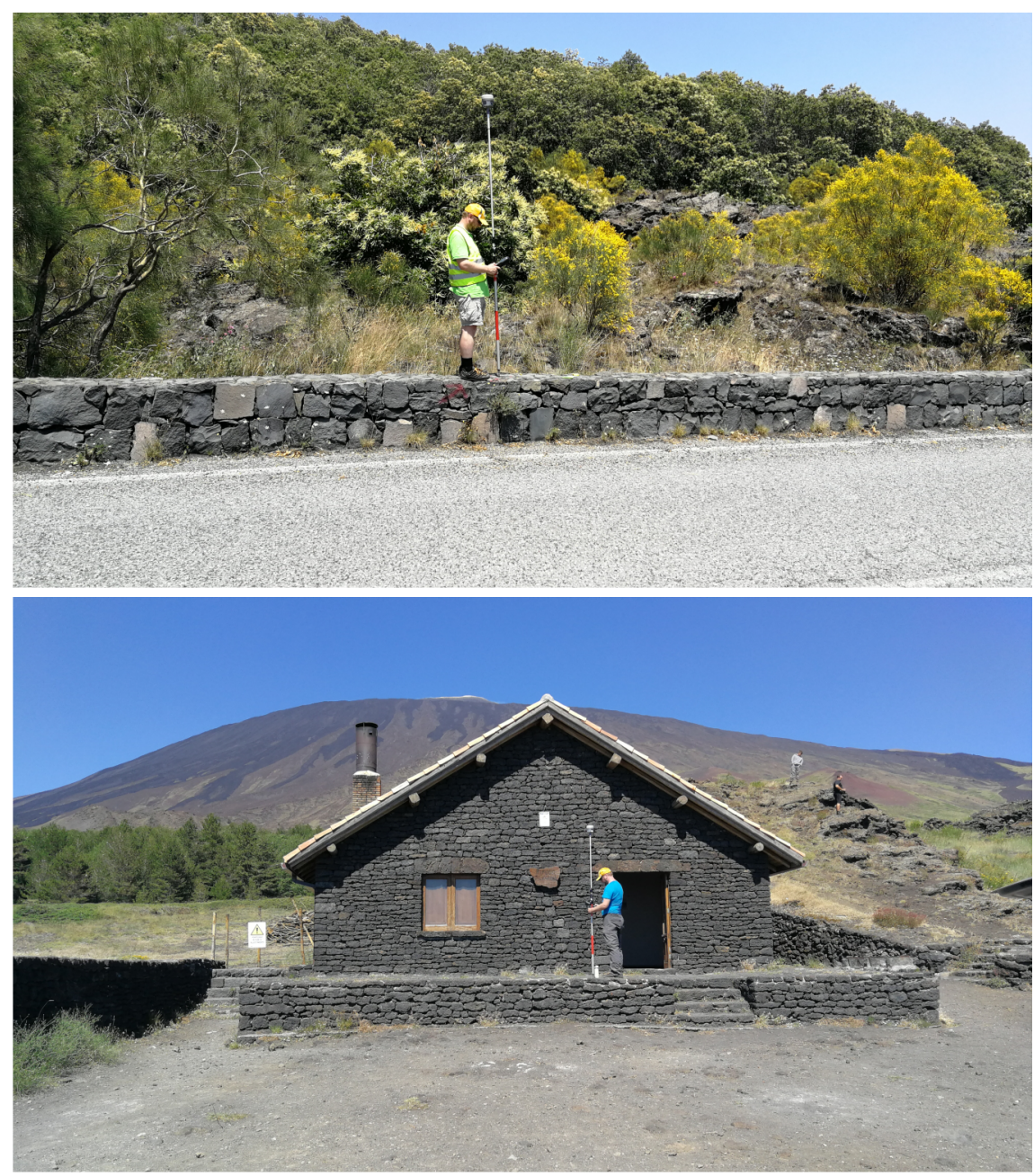

Fig. 5. GNNS measurements on one of relative benchmarks situated on the top of roadside rock-wall (top) and in the front of a refuge with an absolute gravity point inside (bottom).

The absolute gravity points are typically situated inside accessible small refuges (see an example in Fig. 5), except PDN and MNT. PDN is situated inside the basement of the Pizzi Deneri volcanological observatory (managed by INGV Catania). This point is actually underground, which caused the worst VGG prediction (see Fig. 3). MNT is situated inside the build- 
ing of the upper cable-car station. However, this point was not accessible during our campaign. At all absolute gravity points we took the GNSS measurement at an open-air place in their proximity from which we positioned the gravity benchmark inside a building using terrestrial measurements by means of total station. We also measured the dimensions of the buildings and the thickness of their walls, as well as the relative position of benchmark with respect to the building walls (using laser rangefinder), in order to be able to carry out subsequent building correction calculation.

In addition to GNSS/terrestrial geodetic measurements, we also aimed at improving the topographic effect calculation in the nearest zone using droneflown photogrammetry wherever feasible and applicable. Aerial images were taken in the surrounding of the selected absolute/relative benchmarks (diameter of 50 to $120 \mathrm{~m}$ around the point) using the unmanned aerial vehicle - DJI Phantom v. 3 and 4. Minimum six artificial GCPs were prepared and distributed before the flight at each locality. Checked and cleaned stereoscopic images with at least $60 \%$ longitudinal and latitudinal overlap were processed using Agisoft PhotoScan (http://www . agisoft.com/) with standard processing chain into classified point clouds, digital elevation models and georeferenced orthophotomosaics. The quality of the photogrammetric processing was checked using GCPs and independent sets of points and varies between $3-7 \mathrm{~cm}$ in the horizontal position and $5-13 \mathrm{~cm}$ in the height. The automatic classification to the ground class was improved by supervised selection of individual trees, buildings or artefacts (see Fig. 7) in Surfer v. 15. Final digital terrain/surface models with the resolution of $0.5 \mathrm{~m}$ were created in Surfer v. 15 using Kriging interpolation method.

Because of inappropriate environmental conditions (presence of trees, strong wind preventing the drone flight) we managed to perform droneborne photogrammetry only at five benchmarks (see Fig. 2): TG, CV, PDN, GVL and the new point MNT_IG, where the iGrav\# 020 superconducting gravity meter was installed by INGV team in 2016.

We repeated VGG measurements on benchmarks CF and DNV to confirm older measurements (Fig. 3). We made new VGG measurements at two points, LZ and MNT_IG. The VGG measurements were performed in a tower mode using relative gravity meter CG-5 and geodetic tripod (Fig. 6). Gravity acceleration at each point was measured by A-B-A-B-A repeating method at two height levels (with gravity meter sensor approximately $0.25 \mathrm{~m}$ 


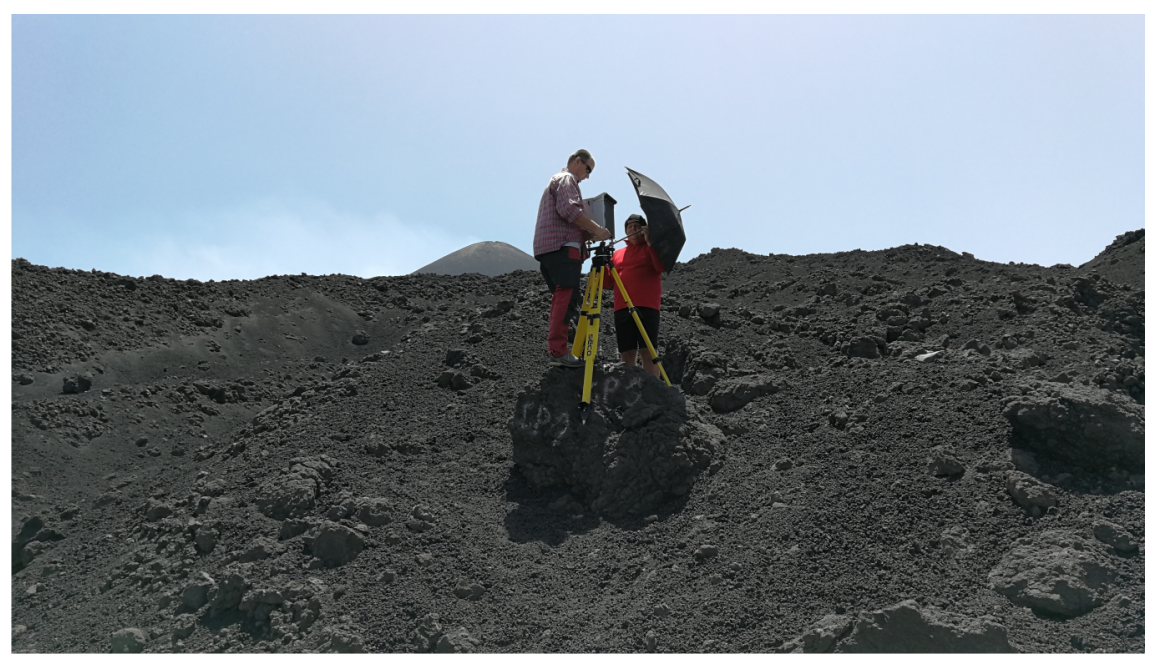

Fig. 6. Tower VGG measurement at point LZ (in its top position) in unusual conditions (with a view of $\mathrm{NE}$ creater in the background). Umbrella had to be used to shield the meter from wind.

(A) and $1.3 \mathrm{~m}$ above the ground (B), depending on the tripod setup). The exact height of the gravity meter sensor above the ground (point/benchmark) was measured using the smart high-precision laser distance meter. Striking wind posed a serious problem for the tower VGG measurement accuracy at several points, especially in the upper measuring position (B) on the tripod. We used a common umbrella (Fig. 6) to shield the gravity meter from the wind, but it was very problematic to get reasonable results in several cases. The strong wind was the reason why we were able to get the VGG at only one of three scheduled points in the NE-rift area. We have estimated a measurement error at particular measurement levels (A and B) of approximately $\pm 7 \mu \mathrm{Gal}$, which leads to an error of about $\pm 10 \mu \mathrm{Gal} / \mathrm{m}$ in VGG.

At the new point MNT_IG, which is situated at the Montagnola hut on the top of an extinct crater (2600 m a.s.l.), we acquired the highest (in absolute sense) VGG value of $-454 \mu \mathrm{Gal} / \mathrm{m}$, which represents a deviation of $47 \%$ from the normal gradient. For a comparison, Greco et al. (2012) reported previously measured gradients on Etna network benchmarks with deviations of up to about $25 \%$. 


\section{Near topography refinement and building corrections}

All in-situ determined data, i.e. accurate horizontal positions and elevations of benchmarks and new measured VGG points (with exact gravity meter sensor heights above the ground), building and roadside wall dimensions, as well as drone-borne photogrammetry-derived DEMs of the nearest zones, were subsequently used to improve the VGG calculation (prediction).

Topographic contribution to the VGG was calculated in the same manner as described previously, using the density of $2670 \mathrm{~kg} / \mathrm{m}^{3}$. Real heights of the gravity meter sensor with respect to the ground were kept in this case. Using exact coordinates and heights of the benchmarks yields an improvement of the topographic contribution in the range from -45 to $+23 \mu \mathrm{Gal} / \mathrm{m}$ (standard deviation of $15 \mu \mathrm{Gal} / \mathrm{m}$ ), while the topographic contributions to the VGG itself ranges from -165 to $+93 \mu \mathrm{Gal} / \mathrm{m}$. The highest negative contribution is at point MNT_IG situated on the top of an extinct crater, the highest positive contribution is at point PDN situated in underground setting. In addition, drone-borne photogrammetric DEMs of the nearest zone were used to improve the innermost zone contribution at five points (within the distance of up to approximately $80 \mathrm{~m}$ ). This improvement is less than $10 \mu \mathrm{Gal} / \mathrm{m}$ at all points except for point PDN, where it reaches $52 \mu \mathrm{Gal} / \mathrm{m}$ due to the fact that the point is located in the basement of the observatory (Fig. 7).

The gravitational effect of building walls, roadside walls, as well as concrete pillar was modeled by means of simple rectangular prisms using the software Potent (Potent, 2010). The real position of the gravity meter sensor with respect to the modeled bodies was accounted for, based on the in-situ measured distances using laser rangefinder. While the geometric definition of the modeled prisms is simple, the estimation of their density is still problematic. We took into account the brick material (basalt) as well as its porosity, so we chose the density of $2500 \mathrm{~kg} / \mathrm{m}^{3}$ for compact walls and $2200 \mathrm{~kg} / \mathrm{m}^{3}$ for the walls made of rocks with higher porosity. In case of the concrete pillar (protruding above floor level) we used the density of $2300 \mathrm{~kg} / \mathrm{m}^{3}$. Station PDN required special attention as it is situated underground. After the calculation of topographic effect (using drone-borne photogrammetric DEM in the innermost zone, see Fig. 7) for a true underground position of the point, we had to also model "empty" underground 


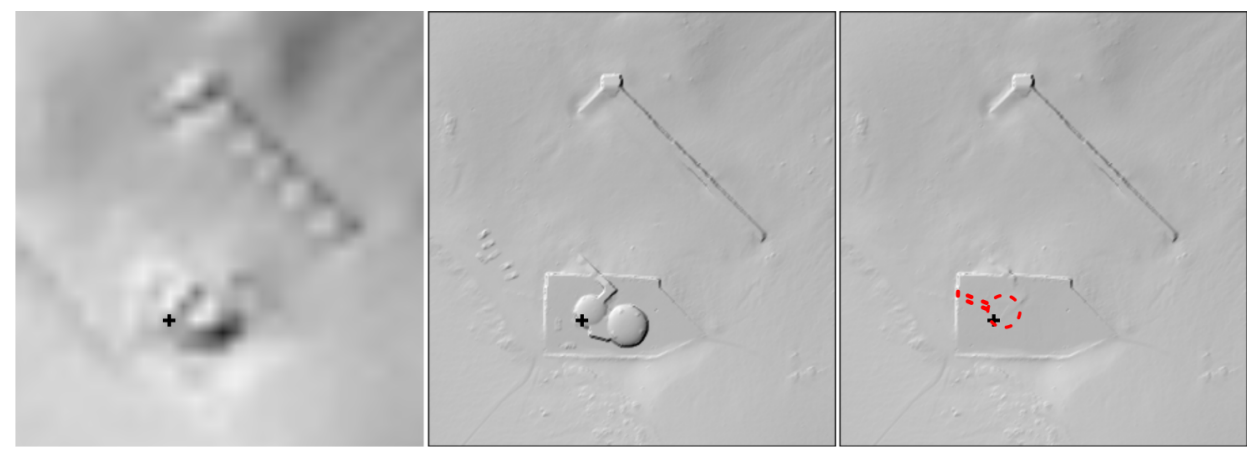

Fig. 7. Comparison of the LiDAR-based DEM (left) with the drone-borne photogrammetric DEM (middle) in the vicinity of the absolute gravity point PDN (black cross) situated in the basement of Pizzi Deneri volcanological observatory (2820 m a.s.l.). The right picture shows the "cleaned" model that was used for the topographic effect calculation. Red dashed line indicates the position of underground basement, which was subsequently also modeled (see Fig. 8).

spaces, see Fig. 8 .

Thus calculated building contributions to the VGG reach -5 to -15 $\mu \mathrm{Gal} / \mathrm{m}$ in case of relative benchmarks situated on roadside walls, and +14 to $+26 \mu \mathrm{Gal} / \mathrm{m}$ in case of absolute gravity points located inside buildings (note that opposite sign of these contributions corresponds to the opposite systematic "shifts" observed from prediction, see Fig. 3). A special case is again PDN, where the "basement" contribution (see Fig. 8) reaches -140 $\mu \mathrm{Gal} / \mathrm{m}$, but this contribution and the topography contribution compensate
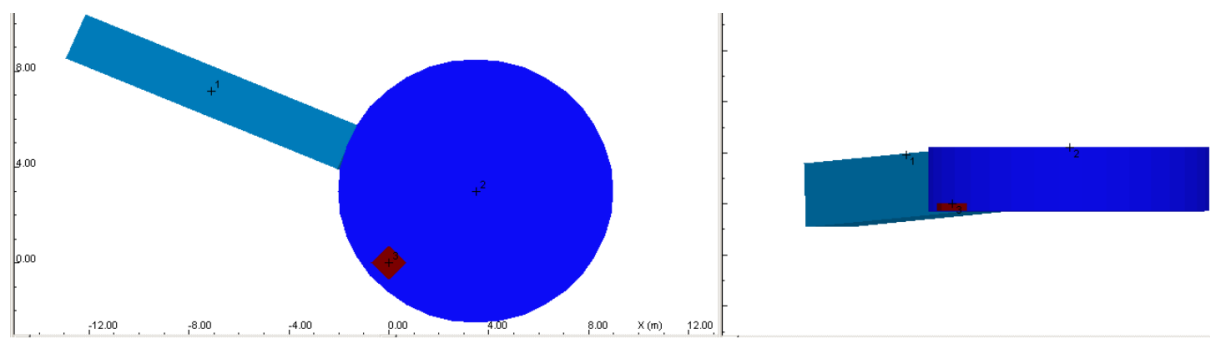

Fig. 8. Modeled underground spaces around point PDN (left - top view, right - side view). Blue model bodies represent basement room with inclined access corridor, small red prism represents a concrete pillar protruding above the floor level. Picture is taken from Potent modeling software interface, axis units are in meters. 
each other to a significant extent.

Since the points GPA and ZAF are located near local terrain steps with a height of approximately two meters, which are not adequately captured by the LiDAR DEM, we used simple prism approximation of these edges to estimate their contribution to the VGG. We estimated these contributions to be +17 and $-8 \mu \mathrm{Gal} / \mathrm{m}$ for GPA and ZAF, respectively (note that opposite sign of these contributions corresponds to opposite field conditions, GPA lies below the terrain edge while ZAF lies on the top of the edge).

Fig. 9 shows comparison between measured VGGs and VGG values calculated (i) using previously available information and (ii) the new information described above (in case of repeated VGG measurements at the same benchmark the portrayed VGG value represents the average values).
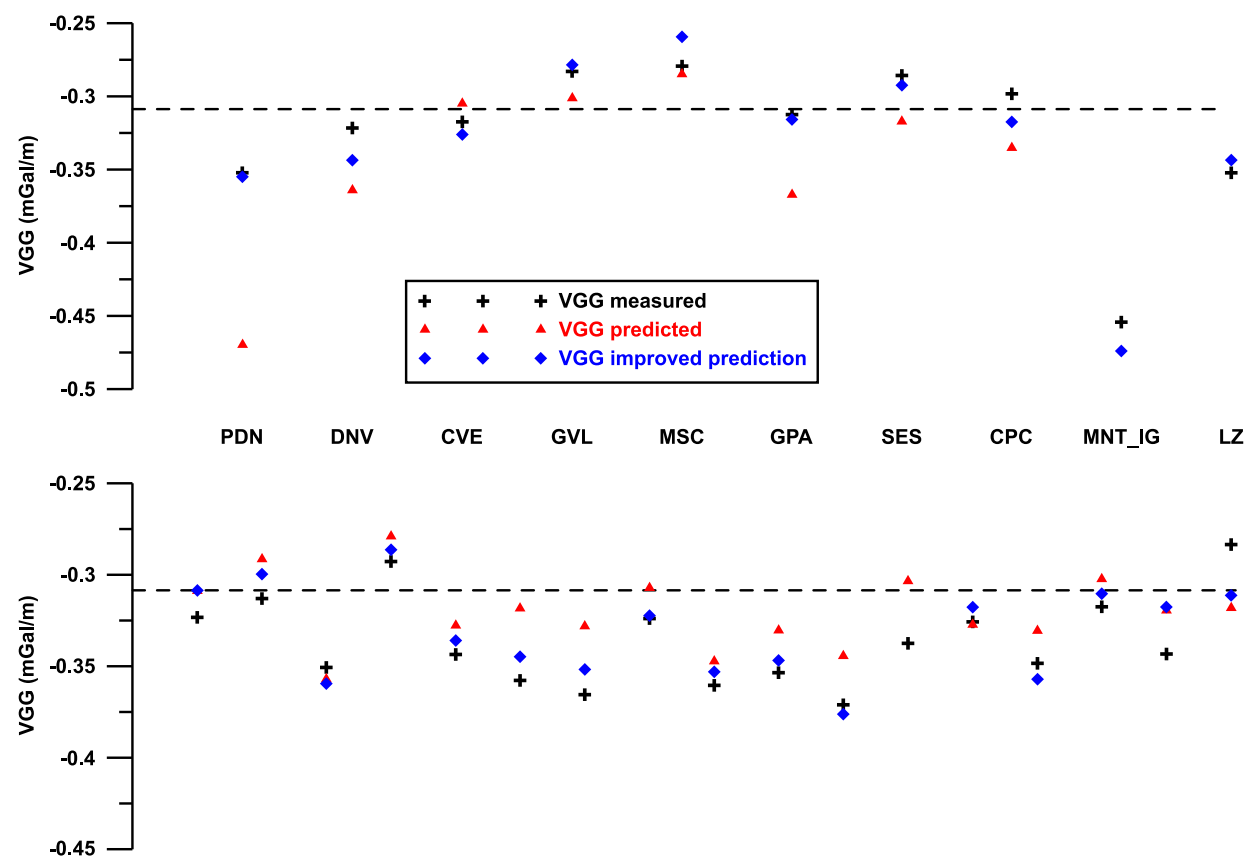

$\begin{array}{lllllllllllllllllllllll}\text { ZAF } & \text { HP } & \text { PT } & \text { BP } & \text { MO } & \text { FV } & \text { CV } & \text { FM } & \text { MS } & \text { RS } & \text { TG } & \text { VE } & \text { IF } & \text { CF } & \text { CE } & \text { CC } & \text { CH }\end{array}$

Fig. 9. Comparison of averaged measured VGGs (black crosses) with previously predicted VGGs (red triangles, also shown in Fig. 3) and newly calculated values (blue diamonds) on absolute gravity points (upper graph) and relative benchmarks (bottom graph). Dashed lines represent theoretical (normal) gradient of $-308.6 \mu \mathrm{Gal} / \mathrm{m}$. 


\section{Discussion}

A relatively good improvement of newly calculated VGGs was achieved in comparison with previously predicted values at most benchmarks (demonstrated by the shift of blue marks closer to the black ones in Fig. 9). Regarding the absolute gravity points (Fig. 9, upper graph), there is a shift towards positive VGG values in most cases, which is mainly the result of introducing the building correction. The most significant improvement has been achieved at PDN, after including the correction for the underground positioning of the gravimeter.

Two newly measured VGG points MNT_IG and LZ are also shown in the mentioned graph. They show a good correlation between measured and calculated VGG values, as they are located outdoor (see Fig. 6), implying that the correction accounting for the hosting building is not needed. At LZ, the difference between the in-situ measured $(-352 \mu \mathrm{Gal} / \mathrm{m})$ and the theoretical $(-308.6 \mu \mathrm{Gal} / \mathrm{m})$ VGG can lead to a significant difference in the free-air correction if large vertical displacements $(>2 \mathrm{~m})$ occur, as in the case of the ground deformation associated with the 2002-2003 NE-rift eruption (Bonforte et al., 2007), cf. Table 1.

Relative benchmarks show an opposite behavior (Fig. 9, bottom graph), as they exhibit a shift towards negative VGG values in most cases. This results from the improved topographic correction, as well as the roadside wall corrections. The correlation between measured and newly calculated VGG values is significantly better at most points compared with previously predicted values, but there are still some points where predictions are not satisfactory, especially points $\mathrm{CC}, \mathrm{CH}$. These points are situated under trees, so it was not possible to perform drone photogrammetry and get more accurate terrain models.

\section{Conclusions}

We can statistically evaluate the improvement of the VGG prediction as follows: standard deviation of differences between measured and predicted VGG values decreased from 39 to $13 \mu \mathrm{Gal} / \mathrm{m}$ at absolute gravity points and from 18 to $12 \mu \mathrm{Gal} / \mathrm{m}$ in case of relative benchmarks. In terms of maximum 
differences (in absolute sense) we got improvement from 117 to $22 \mu \mathrm{Gal} / \mathrm{m}$ (absolute points) and from 39 to $28 \mu \mathrm{Gal} / \mathrm{m}$ (relative points). Regarding the relative importance of the different contributions to VGGs, we have to distinguish between "surface" benchmarks and the underground PDN point. At PDN, an important contribution to VGG comes from considering the empty underground spaces. Furthermore, drone-borne photogrammetry allowed to improve the topographic contribution of the inner-zone (a difference of $52 \mu \mathrm{Gal} / \mathrm{m}$ was found for this component). Conversely, the inner-zone topographic contribution improvement was relatively small at other points (less than $10 \mu \mathrm{Gal} / \mathrm{m}$ ). In the case of absolute gravity points, the correction accounting for the building that hosts the gravimeter reaches $26 \mu \mathrm{Gal} / \mathrm{m}$ in maximum. Finally, the accurate geodetic re-positioning of benchmarks resulted in an improvement in the topographic contribution of up to $45 \mu \mathrm{Gal} / \mathrm{m}$. These results indicate that accurate VGG predictions require precise information on topography and point positioning, as well as consideration of the contribution from man-made structures in close proximity to the observation point.

Acknowledgements. This work was supported by the VEGA grant agency under projects No. $2 / 0042 / 15$ and $1 / 0462 / 16$ as well as by the Slovak Research and Development Agency, grant No. APVV-16-0482 (acronym Lithores).

\section{References}

Battaglia M., Gottsmann J., Carbone D., Fernández J., 2008: 4D volcano gravimetry. Geophysics 73, 6, WA3-WA18, doi: 10.1190/1.2977792.

Bisson M., Spinetti C., Neri M., Bonforte A., 2015: Mt. Etna volcano high-resolution topography: airborne LiDAR modelling validated by GPS data. International Journal of Digital Earth, doi : 10.1080/17538947.2015.1119208.

Bonforte A., Carbone D., Greco F., Palano M., 2007: Intrusive mechanism of the 2002 NErift eruption at Mt. Etna (Italy) modelled using GPS and gravity data. Geophys. J. Int., 169, 339-347, doi: 10.1111/j.1365-246X.2006.03249.x.

Bonforte A., Fanizza G., Greco F., Matera A., Sulpizio R., 2017: Long-term dynamics across a volcanic rift: 21 years of microgravity and GPS observations on the southern flank of Mt. Etna volcano. J. Volcanol. Geotherm. Res., 344, 174-184.

Branca S., Coltelli M., Groppelli G., Lentini F., 2011: Geological map of Etna volcano, 1:50,000 scale. Ital. J. Geosci. (Boll. Soc. Geol. It.), 130, 3, 265-291, doi : 10.3301/ IJG.2011.15. 
Budetta G., Grimaldi M., Luongo G., 1989: Variazioni di gravit'a nell'area etnea (19861989). Boll. GNV, 5, 137-146 (in Italian).

Carbone D., Greco F., 2007: Review of Microgravity Observations at Mt. Etna: A powerful Tool to Monitor and Study Active Volcanos. Pure Appl. Geophys., 164, 769-790, doi : 10.1007/s00024-007-0194-7.

Carbone D., Poland M. P., Diament M., Greco F., 2017: The added value of time-variable microgravimetry to the understanding of how volcanoes work. Earth-Science Reviews 169, 146-179, doi: 10.1016/j.earscirev.2017.04.014.

Fernández J., Pepe A., Poland M. P., Sigmundsson F., 2017: Volcano Geodesy: Recent developments and future challenges. J. Volcanol. Geotherm. Res., 344, 1-12.

Greco F., Currenti G., D’Agostino G., Germak A., Napoli R., Pistorio A., Del Negro C., 2012: Combining relative and absolute gravity measurements to enhance volcano monitoring. Bull. Volcanol., 74, 1745-1756.

Jarvis A., Reuter H. I., Nelson A., Guevara E., 2008: Hole-filled SRTM for the globe Version 4, available from the CGIAR-CSI SRTM 90m Database: http://srtm.csi.cgi ar.org.

Maucourant S., Giammanco S., Greco F., Dorizon S., Del Negro C., 2014: Geophysical and geochemical methods applied to investigate fissures-related hydrothermal systems on the summit area of Mt. Etna volcano. J. Volcanol. Geotherm. Res., 280, 111-125, doi: $10.1016 / j \cdot j$ volgeores.2014.05.014.

Olson Ch. J., Becker J. J., Sandwell D. T., 2016: SRTM15_PLUS: Data fusion of Shuttle Radar Topography Mission (SRTM) land topography with measured and estimated seafloor topography (NCEI Accession 0150537). Version 1.1. NOAA National Centers for Environmental Information.

Palano M., Rossi M., Cannavò F., Bruno V., Aloisi M., Pellegrino D., Pulvirenti M., Siligato G., Matiia M., 2010: Etn@ref: a geodetic reference frame for Mt. Etna GPS networks. Annals of Geophysics, 53, 4, doi: 10.4401/ag-4879.

Pavlis N. K., Holmes S. A., Kenyon S. C., Factor J. K., 2012: The development and evaluation of the Earth Gravitational Model 2008 (EGM2008). Journal of Geophysical Research: Solid Earth, 117, B04406: http://dx.doi.org/10.1029/2011JB008916.

Potent v.4.11.06, 2010: User guide. Manuscript, Geophysical Software Solutions Pty. Ltd., Gungahlin, Australia.

Schiavone D., Loddo M., 2007: 3-D density model of Mt. Etna Volcano (Southern Italy). Journal of Volcanology and Geothermal Research, 164, 161-175.

Vajda P., Zahorec P., Papčo J., Kubová A., 2015: Deformation induced topographic effects in inversion of temporal gravity changes. Contributions to Geophysics and Geodesy, 45, 2, 149-171.

Zahorec P., Papčo J., Mikolaj M., Pašteka R., Szalaiová V., 2014: The role of near topography and building effects in vertical gravity gradients approximation. First Break,32, 1, 65-71.

Zahorec P., Vajda P., Papčo J., Sainz-Maza S., Pereda de Pablo J., 2016: Prediction of vertical gradient of gravity and its significanse for volcano monitoring - example from Teide volcano. Contributions to Geophysics and Geodesy, 46, 3, 203-220. 
Zahorec P., Marušiak I., Mikuška J., Pašteka R., Papčo J., 2017: Numerical Calculation of Terrain Correction Within the Boguer Anomaly Evaluation (Program Toposk), chapter 5, 79-92, In book: Roman Pašteka, Ján Mikuška and Bruno Meurers (Eds.): Understanding the Bouguer Anomaly: A Gravimetry Puzzle, Elsevier, ISBN 978-012-812913-5, doi : 10.1016/B978-0-12-812913-5.00006-3. 